\title{
Mendeley Mobile: Powerful Cloud-Based Article and Reference Management in Your Pocket
}

\author{
James Shin ${ }^{1}$
}

Published online: 27 September 2016

(C) Society for Imaging Informatics in Medicine 2016

\author{
App Specs \\ App Icon URL: https://lh3.googleusercontent.com/KdvN1 \\ UJWNw6sB1H80T3RO01SM0OmDR1li_vq9ShVEL6 \\ SAoekKVmAPAoQGgfF8CFrCtc=w300 \\ App Name: Mendeley \\ App Developer: Mendeley Ltd. \\ App Developer Website: http://www.mendeley.com \\ App Price: FREE (premium storage upgrade options) \\ Apple App Store URL: https://itunes.apple. \\ com/us/app/mendeley-pdf-reader/id380669300?mt=8 \\ Google Play Store URL: https://play.google. \\ $\mathrm{com} /$ store/apps/details?id=com.mendeley\&hl=en \\ Category: journal, educational, reference, clinical, research \\ Tags: \#research, \#PDF, \#organizer, \#cloud, \#iOS, \#an- \\ droid, \#works-offline, \#free, \#metadata \\ Works Offline: [Y] \\ FDA Approval: [NA] \\ Promotion Code:
}

\section{Quick Review}

(1 star: lowest/5 stars: highest)

Overall Rating (1-5): 5

Content (1-5): 5

Usability (1-5): 5

Pros: Free 2GB cloud-based database with offline PDF viewing and annotation syncing capabilities. Integrates with desktop client to provide full-featured citation management, article metadata matching, and library organizing tools.

James Shin

jjs7005@med.cornell.edu

1 Department of Radiology, Weill Cornell Medical College, 525 E 68th St., New York, NY 10065, USA
Cons: No integrated Pubmed search engine. Lack of direct web import option for Android devices (iOS Safari bookmarklet available).

At a Glance: Free 2GB cloud-based article management system for platform-independent access to personal collection of research publications. Database abstracts and article metadata are searchable, with downloadable full-text PDFs for offline viewing on $\mathrm{iOS}$ and Android devices. PDFs can be highlighted and annotated, and changes propagate to the cloud when online. Integrates with desktop client which includes citation manager and automated library management system with customizable file/folder tree naming schemes. Browserspecific extensions for one-click importing from any online computer are very useful and integrate with automated article metadata matching.

\section{Full Review}

Intro: Information management is a critical skill to master to successfully wade through increasingly vast bodies of knowledge available for consumption. A multitude of information management tools and services exist aimed at increasing the time efficiency of clinical research, education, and publication, and Mendeley is one such tool.

Purpose/Features/Content:

Mendeley is a cloud-based article management system accessible via a multitude of mobile, web, and desktop clients. Users may register for a free account, which includes $2 \mathrm{~GB}$ of cloud storage for centralized access to collected publications. Mobile apps are available for iOS and Android devices, with keyword searchable article abstract and metadata. Available full-text PDF's may be downloaded for offline viewing and annotation (text and highlight), with changes propagating to the cloud when online.

Usability: Mobile app provides a functional PDF reader with basic annotation tools and facile email sharing. Article 
metadata can be edited within the app; however, article matching is only available via web or desktop client. Direct importing via $\mathrm{PC} / \mathrm{Mac}$ browser extension or iOS Safari bookmarklet is available for PubMed, IEEE Xplore, Google Scholar, PLoS, Elsevier, Scopus, and ScienceDirect, among others.

Good: Free and exceptionally functional.

Screenshots (Minimum of Two; Figs. 1, 2, 3, and 4)

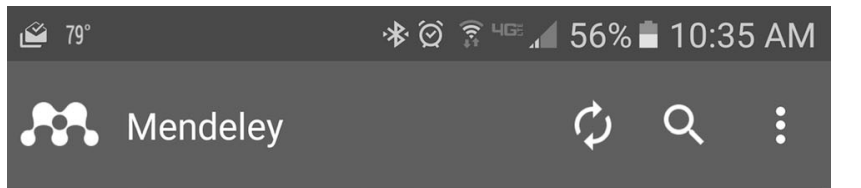

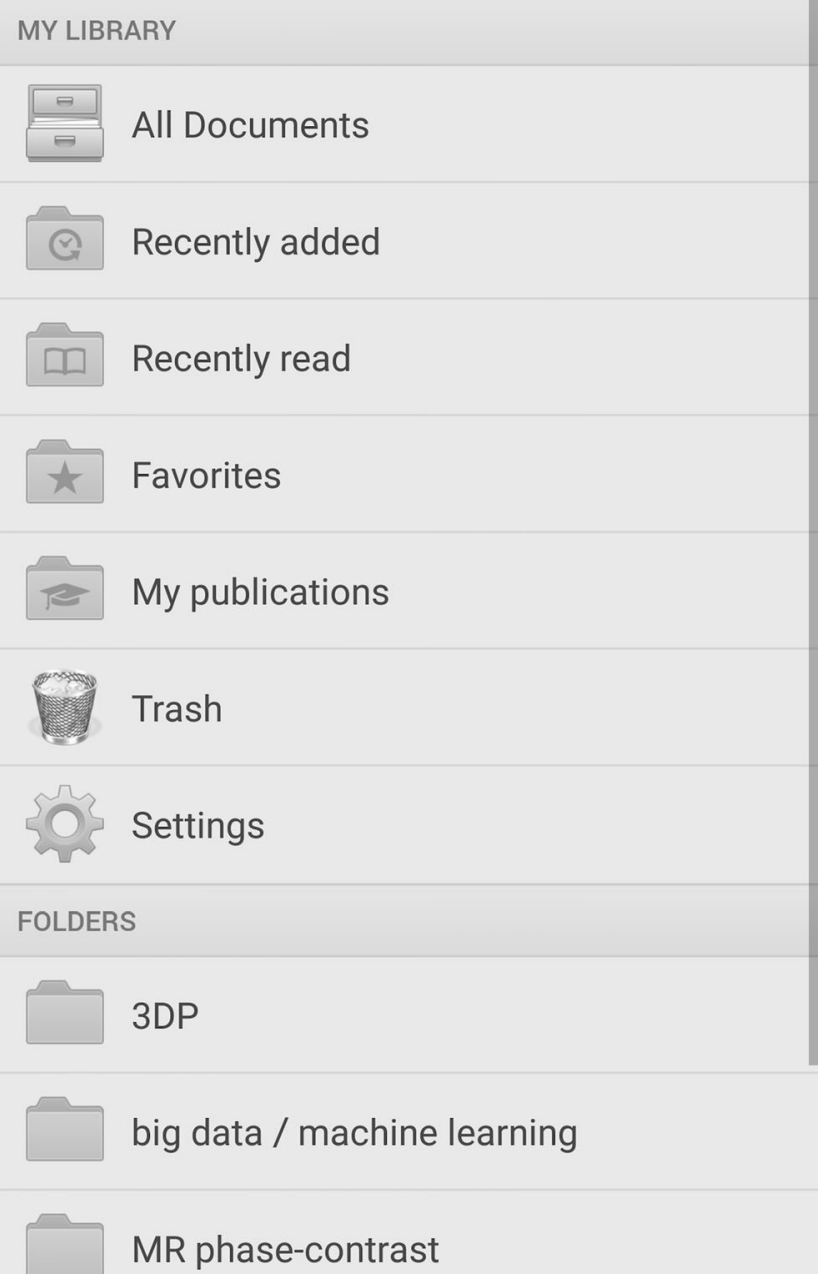

Fig. 1 Main menu
Room for Improvement: Overall an excellent piece of software. Integrated in-program Pubmed search could be useful on the mobile app, although this is a feature more sorely missing from the desktop client. Lack of web importing option for Android devices is notable, however, does not substantially diminish the utility of the app even on Android devices.

\section{ABSTRACT}

Large skull defects as a result of craniectomies due to cerebral insults, trauma, or tumors create functional and aesthetic disturbances for the patient. Cranioplasty with implants in these cases are an alternative to autogenous bone transplantation.

\section{TAGS}

Add...

\section{$\square$ Read article}

Fig. 2 Article preview 


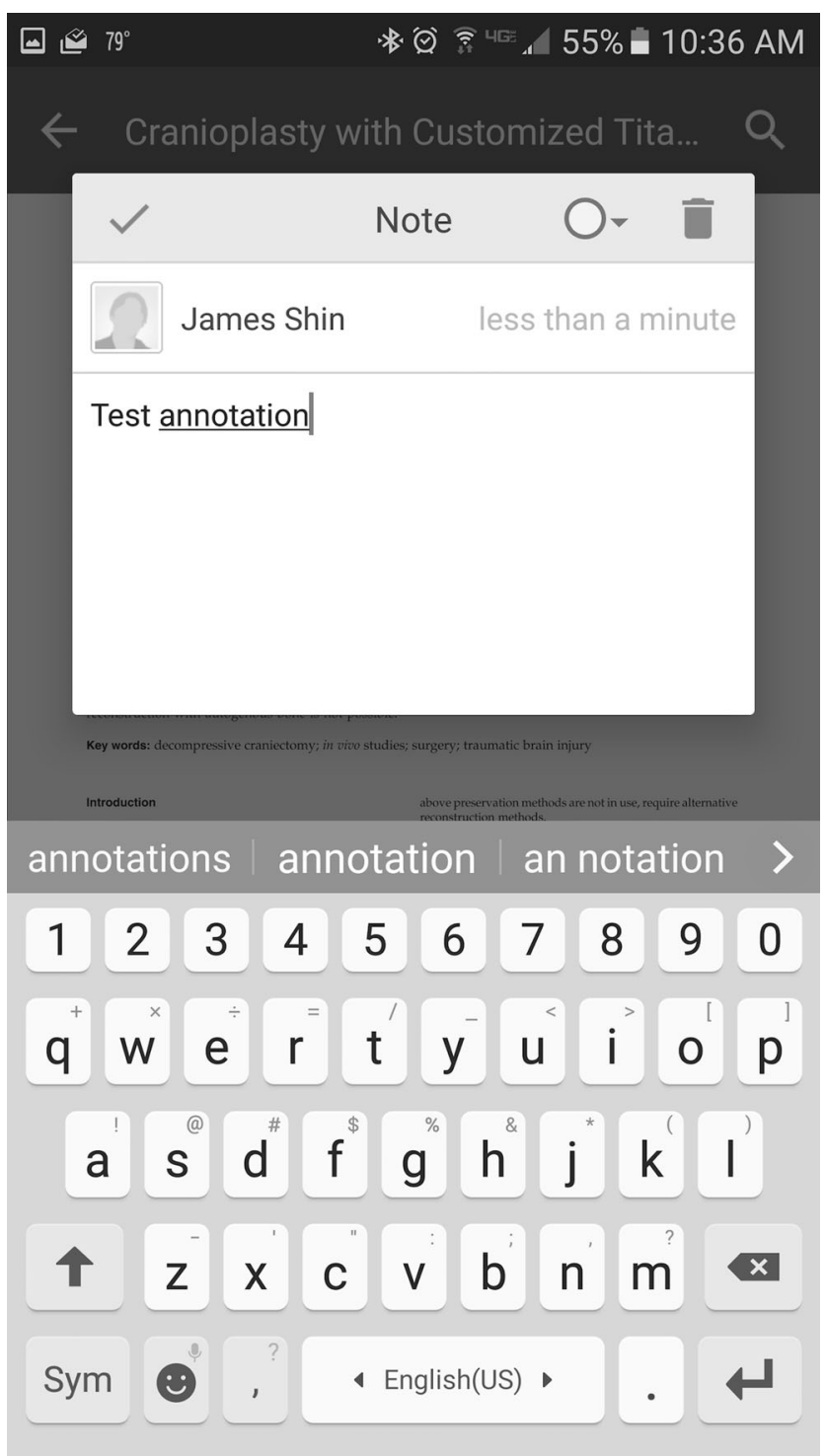

Fig. 3 Creating PDF annotations

$\leftarrow \quad$ Cranioplasty with Customized Tita...

Q

JOURNAL OF NEUROTRAUMA 29:1077-1083 (April 10, 2012)

(c) Mary Ann Liebert, Inc.

\section{三तnioplasty with Customized and PEEK Implants in a Mechanica}

Bernd Lethaus ${ }^{1}$ Yara Safi, ${ }^{2}$ Mariel ter Laak-Poort ${ }^{3}$ Anita Kloss-B Christian Robbenmenke, ${ }^{2}$ Ulrich Steinseifer, ${ }^{2}$ and $\mathrm{P}$

\section{Abstract}

Large skull defects as a result of craniectomies due to cerebral insults, trau aesthetic disturbances for the patient. Cranioplasty with implants in these $\mathrm{c}$ bone transplantation. In our clinic, customized titanium or optima poly-eth used to reconstruct craniectomy defects. To compare the two materials we i the implants fixed to a sintered polyamide skull model under mechanical s standard testing machine, the models were subjected to a load under a quasiFractures of the PEEK implants occurred at a force of 24.2 and $24.5 \mathrm{kN}$ with titanium implants showed no deformation, but extensive damage was seen highest pressures achieved were 45.8 and $50.9 \mathrm{kN}$. In a simplified model wit withstood forces that were higher than those capable of causing skull fra properties of PEEK could provide better protection when used for craniop reconstruction with autogenous bone is not possible.

Key words: decompressive craniectomy; in vivo studies; surgery; traumatic

Introduction

DCOMPRESSIVE CRANIECTOMY to decrease intracranial pressure after brain infarction, intracranial hemorpre or cerebral edema is an established procedure in neurosurgery. This procedure exposes parts of the brain, levin it unprotected and giving the pationt a malform it unpretect a cang ance. Cranioplasty car restore the ase the appearance and offers mech beal proction in case of trapp. The autogenous bone is regarded in reconstructive bone surgery as the gold standard to re-establish the cranium's integrity

The resected bone flap can be stored in a bone bank, which is not always available in every neurosurgical center. Another

Fig. 4 Cloud syncing of local PDF annotations

above preservation $\mathrm{r}$ reconstruction meth Defects can also $t$ donor sites. Howeve size and applies on customized or nonautogenous br titanium, various pli different marious (a) $1999 ;$, Wilting al, Wiltfang et al., 2002 Titanium and PEF customized implant 